\title{
Seismic-Source Corner Frequencies from the Depth of Burial Experiment
}

\author{
Marvin D. Denny
}

November 23, 1998

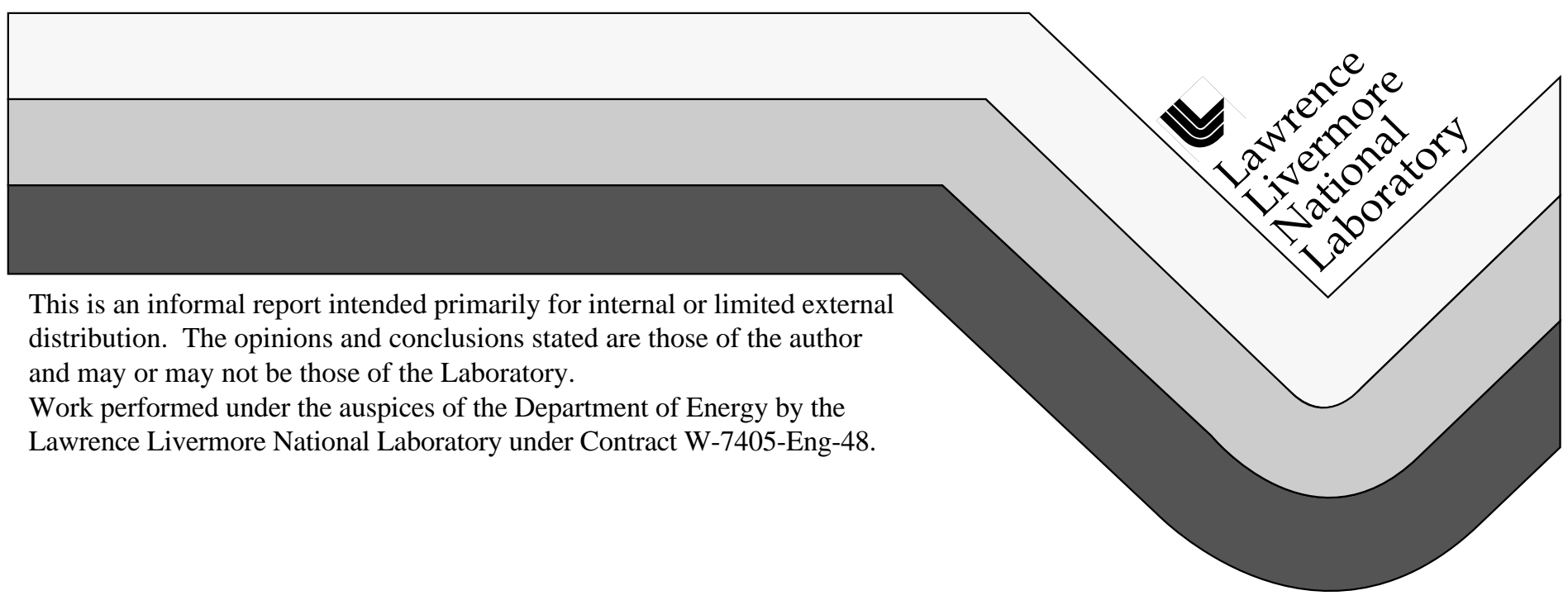




\section{DISCLAIMER}

This document was prepared as an account of work sponsored by an agency of the United States Government. Neither the United States Government nor the University of California nor any of their employees, makes any warranty, express or implied, or assumes any legal liability or responsibility for the accuracy, completeness, or usefulness of any information, apparatus, product, or process disclosed, or represents that its use would not infringe privately owned rights. Reference herein to any specific commercial product, process, or service by trade name, trademark, manufacturer, or otherwise, does not necessarily constitute or imply its endorsement, recommendation, or favoring by the United States Government or the University of California. The views and opinions of authors expressed herein do not necessarily state or reflect those of the United States Government or the University of California, and shall not be used for advertising or product endorsement purposes.

This report has been reproduced directly from the best available copy.

Available to DOE and DOE contractors from the Office of Scientific and Technical Information

$$
\text { P.O. Box 62, Oak Ridge, TN } 37831
$$

Prices available from (615) 576-8401, FTS 626-8401

Available to the public from the National Technical Information Service

U.S. Department of Commerce 5285 Port Royal Rd. Springfield, VA 22161 


\title{
Seismic-Source Corner Frequencies \\ from the \\ Depth of Burial Experiment
}

\begin{abstract}
The results from the depth of burial experiment (DOB) are consistent with cuberoot scaling and with previous observations that the source corner frequency for underground explosions increases with depth. The corner frequencies, however, were overpredicted by Mueller and Murphy (1971) and underpredicted by Denny and Johnson (1991).
\end{abstract}

\section{Introduction}

The depth of burial (DOB) experiment was conducted at the former Soviet nuclear test site in Kazakstan (Phillips et. al., 1998). It was intended to validate empirical observations that the source corner frequency of an underground explosion increases with depth of burial whereas the seismic moment decreases. These dependencies were first documented by Mueller and Murphy (1971), and later by Denny and Johnson (1991) though their detailed descriptions differ. The former assumed a scaling law for amplitudes and yields, and the latter determined it by regression analysis. Skeptics have questioned both analyses-in the first case, by questioning scaling law, and in the second case, by questioning the validity of the regression analysis. For operational safety, the nuclear test program always buried a test according to its expected yield. Thus, the validity of the regression analysis depended on the actual yield being significantly different from the planned yield. Although this requirement was met in a statistical sense, in the eyes of many, this was not sufficient. The DOB experiment was meant to resolve these doubts by detonating the same amount of explosive at significantly different depths. It was also meant to shed some light on the question of overshoot, or damping, in the seismic-source function.

\section{Background}

The simplest model of an explosion was given by Sharpe (1942). He described it as a radial stress applied to a spherical surface whose radius $R$ is large enough that the motion beyond it is determined by linear elastic theory. For the case where stress $P_{0}$ is a step function, the LaPlace transform of the source function of the resulting equation of motion, as given in Denny-Johnson, is

$\psi(s)=\frac{P_{0} R^{3} \omega_{e}^{2}}{4 \mu \mathrm{s}\left(s^{2}+2 \eta \omega_{e} s+\omega_{e}^{2}\right)}$,

where $\mu$ is the shear modulus, $\eta$ is a damping factor determined by the ratio of the shear and compressional wave speeds, and $\omega_{e}$ is the corner frequency in radians given by $\omega_{e}=2 \beta / R$, where $\beta$ is the shear velocity. If the elastic radius $R$ scales as the cube-root of the yield, $R \propto W^{1 / 3}$, then the source function scales as the yield to the first power.

Equation (1) cannot be evaluated experimentally without detailed knowledge of the propagation paths through the real earth from the source to a recording site. However, if two explosions are fired at the same place and recorded at the same sites, then all of the complicated propagation factors cancel when the ratio between the two signals is taken. To be meaningful, however, the two sources must be significantly different in size or the ratio is nearly a constant. Writing Eq. (1) as the ratio of a large explosion $l$ to a smaller calibration explosion $c$ and assuming cube-root scaling, we have

$$
\frac{\psi_{l}}{\psi_{c}}=\frac{W_{l}}{W_{c}} \frac{\omega_{l}^{2}\left(s^{2}+2 \eta \omega_{c} s+\omega_{c}^{2}\right)}{\omega_{c}^{2}\left(s^{2}+2 \eta \omega_{l} s+\omega_{l}^{2}\right)} .
$$

At low frequencies, i.e., less than $\omega_{l}$, Eq. (2) asymptotically approaches the ratio of the yields, and at high frequencies, it tends to the cube-root of the ratio of the yields. 
In the DOB experiment, three pairs of large and small explosions were detonated at 50, 300, and $550 \mathrm{~m}$ in hard rock. Each calibration shot was located at the midpoint of the subsequent 25ton shot, and all pairs were recorded on the surface by two or more three-component seismic stations at distances of 4 to $17 \mathrm{~km}$ (Phillips et. al., 1998). The calibration shots varied slightly in size with the 50-, 300-, and 550-m events being 92, 50 , and $107 \mathrm{~kg}$, respectively. The large shots, however, were all 25 tons. From Eq. (2) then, the low-frequency asymptotes were expected to be 274, 488, and 234 for the 50-, 300-, and 550-m pairs, respectively; whereas, the high-frequency asymptotes were expected to be 6.5, 7.9, and 6.2. The ratio between the corner frequencies (i.e., of the calibration shot to the 25-ton shot) is the same as between the high-frequency asymptotes.

\section{Method of Analysis}

The data were more difficult to interpret than anticipated. The plan was to first compute the smoothed signal-to-noise ratio for each signal and the spectral ratios for each of the pairs. From these, the useful frequency range of the data would be determined and a scaling relationship established from the low-frequency asymptote. Then the corner frequencies would be computed using the pole-zero estimation procedures previously used to analyze the Salmon-Sterling decoupling experiment (Denny and Goodman, 1990). However, the spectral ratios were not as well behaved as those in the Salmon-Sterling case, and for some signals, this had an adverse effect on the results obtained by the pole-zero analysis. Consequently, we employed another simpler procedure to augment and validate the pole-zero results.

\section{Spectral Ratios and Signal-to-Noise Ratios}

In general, the spectral ratios were consistent with cube-root scaling and with the previous work that showed that the corner frequency increases with depth of burial. These ratios also showed a decay, or roll-off, above the corner frequency of the 25-ton shots, which is consistent with Eq. (2). However, without exception, the high-frequency asymptote and the corner frequency of the calibration shots could not be estimated because of system or gauge noise. Figure 1 illustrates these observations. The spectral ratios shown are for station 8 and the $50-\mathrm{m}$ pair of shots. The apparent corner is about $8 \mathrm{~Hz}$ for all three components, whereas the low-frequency asymptote ranges from 200 on the tangential component to about 400 on the radial component. The corresponding corner frequency of the calibration event should be about $50 \mathrm{~Hz}$; however, the ratios deteriorate at about this value. For the other 25-ton events, the corner frequencies were clearly above $20 \mathrm{~Hz}$, making the corner frequencies of the calibration events close to the folding frequency. Consequently, in all cases, the corner frequency of the calibration shots could not be determined.

Although the spectral ratios typically deteriorated somewhere around $50 \mathrm{~Hz}$, the signal-tonoise ratios generally were greater than 2 at frequencies up 80 or $120 \mathrm{~Hz}$ and even greater in a few cases. However, the data are not fully useful over this range because of what may be a mechanical problem with the seismometers. This is best illustrated by the tangential component of station 7 and the data from the $50-\mathrm{m}$ pair of events as shown in Fig. 2. Here, the spectra are seen to be decaying smoothly above $10 \mathrm{~Hz}$, but at about 40 $\mathrm{Hz}$, both abruptly dip down and then jump back

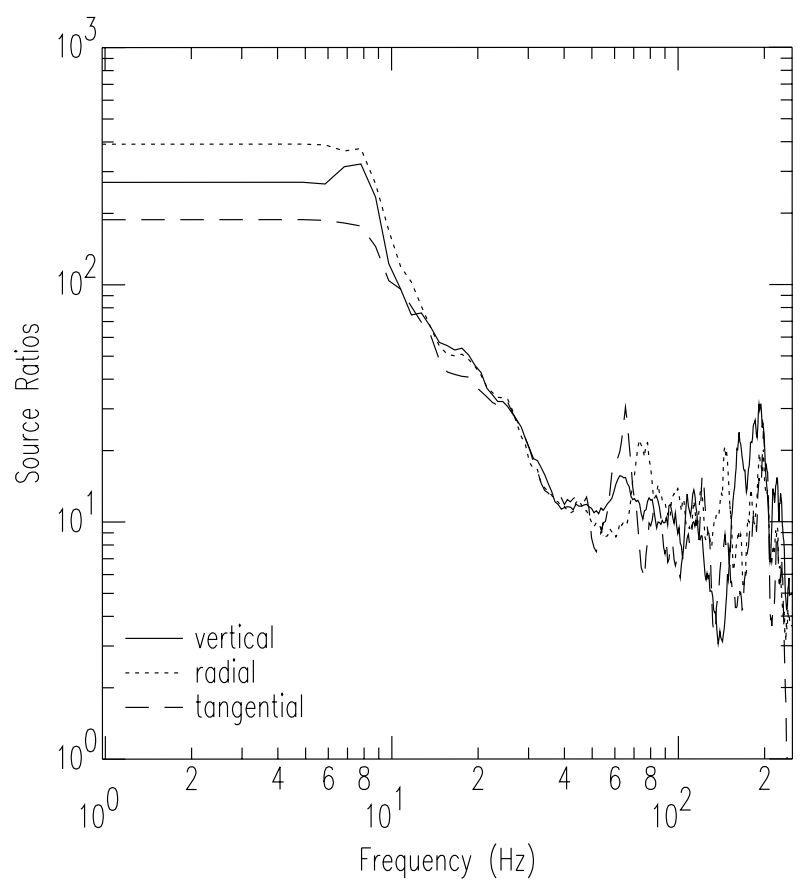

Figure 1. Spectral ratios for the 50-m experiment at station 8 . The spectral ratios were, in general, consistent with cube-root scaling and Sharpe's model. The lowfrequency asymptotes bracket the theoretical value of 274. The corner frequency of the calibration shot should be about $50 \mathrm{~Hz}$; however, neither it nor the highfrequency asymptote can be resolved since the ratios all deteriorate above $40 \mathrm{~Hz}$. The signal length used in these estimates was $0.65 \mathrm{sec}$, and the smoothing operator was 11 points in length. 
up. The jump up could be due to a $60-\mathrm{Hz}$ spike in the electrical power supply; however, the sharp dip is probably due to a suspension problem in the spring-mass system of the seismometer itself. It is present to some degree in all of the signals and reduces the coherency between the signals from the 25-ton and calibration shots.

\section{Pole-Zero Analysis}

Equation (2) is the ratio of two polynomials, each of which has a pair of zeroes. In terms of Shape's model, these are complex pairs that define a corner frequency and a damping factor. In the case of the denominator, the zeroes are also known as poles. Thus, the poles of Eq. (2) define the corner frequency and damping for the 25-ton shot, and the zeroes define the corner frequency and damping of the calibration shot. However, as discussed above, the zeroes cannot be resolved from the experimental data. Fortunately, the theoretical separation between the corner frequencies of the two shots is sufficiently great that the calibration shot can be considered to be an impulsive source with a constant, flat spectrum. In this case, Eq. (2) can be viewed as a low-pass

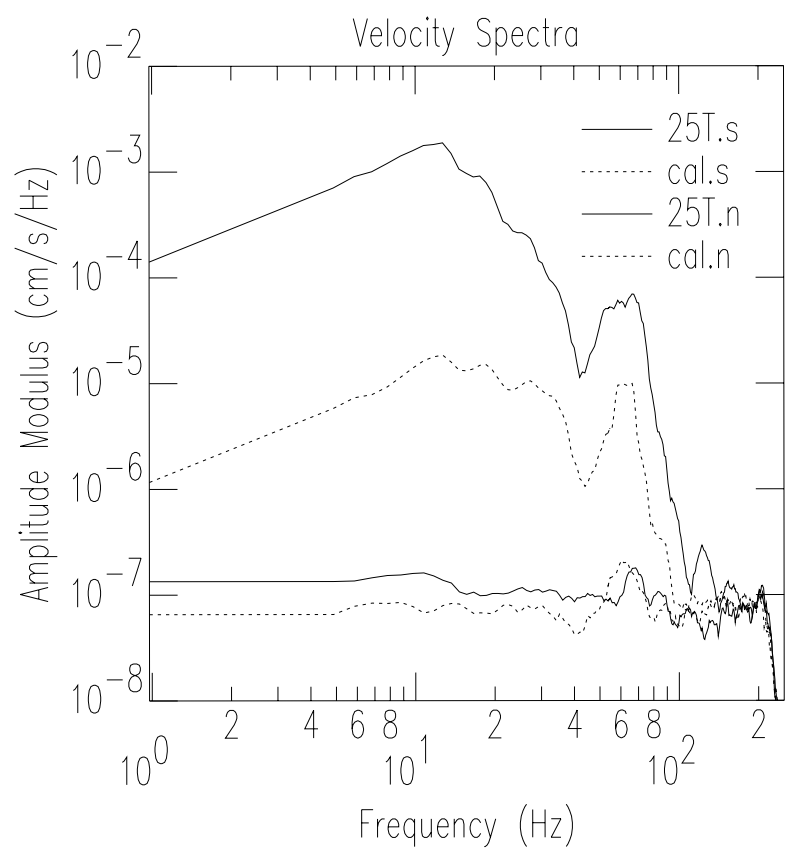

filter that transforms the calibration event into the 25-ton event.

\section{Goodman Method}

The optimal low-pass filter parameters were determined for each pair of signals using the technique of Goodman (1983), and the results are given in Table 1. Ideally, the gain, corner frequency, and damping could be determined for all pairs. In practice, however, the technique may not converge to a solution or, even when it does, one of two problems can occur depending on the quality of the signals. In one case, the optimal solution may consist of two real poles instead of a complex pair. In the other case, a high level of noise may result in a solution with significant ringing, or underdamping (i.e., $\eta<0.7$ ), and a low gain factor. These two types of behavior are illustrated in Fig. 3. A case of two real poles is shown in Fig. 3a, and a case of underdamping is shown in Fig. 3b. In the first case, the gain, or low-frequency asymptote, is unaffected by the fact that the noise causes the method to produce two real poles instead of a complex pair. In the second case, however, the gain is affected by the

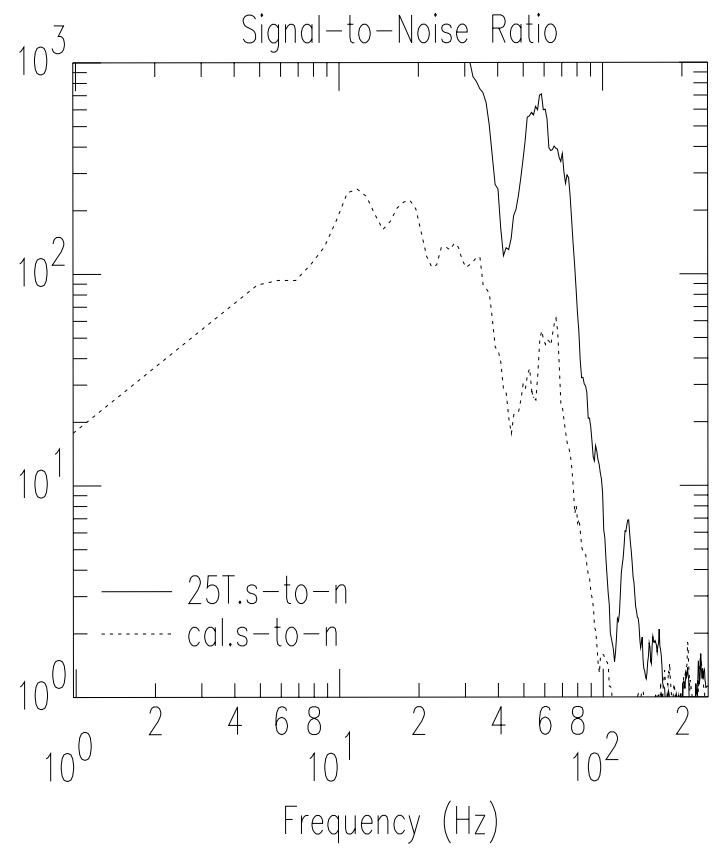

Figure 2. Velocity spectrum and seismometer response. All the spectral ratios deteriorate at about $40 \mathrm{~Hz}$ as in the example shown in Fig. 1 even through the signal-to-noise ratio is greater than 2 up to frequencies as high as $120 \mathrm{~Hz}$ in some cases. The reasons for this may be a structural resonance problem in the seismometers. In this case (50-m $\mathrm{DOB}$, station 7, tangential component), the signal-to-noise ratio is excellent up to $100 \mathrm{~Hz}$ but the spectra both have a huge notch in them between 40 and $50 \mathrm{~Hz}$. If this were due to some wave propagation phenomenon, it would be coherent and not be a problem. As it is, this source of noise distorts many of parameter estimates. 
fact the method produced an underdamped, complex pair. This can be seen in Fig. 3 by comparing the model transfer functions with the data. The models are based on the expected lowfrequency ratios and the results given in Table 1. In both cases, they give a good representation of the data.
Table 1. Corner frequency and damping estimates.

\begin{tabular}{ccccc}
\hline $\begin{array}{c}\text { Depth } \\
(\mathbf{m})\end{array}$ & $\begin{array}{c}\boldsymbol{f}_{\mathbf{0}} \\
(\mathbf{H z})\end{array}$ & $\eta$ & $\begin{array}{c}\text { No. of } \\
\text { stations }\end{array}$ & $\begin{array}{c}\text { No. of } \\
\text { components }\end{array}$ \\
\hline \hline 50 & $9.5 \pm 0.7$ & $0.8 \pm 0.2$ & 2 & 4 \\
300 & $23.8 \pm 3.2$ & $0.6 \pm 0.2$ & 4 & 9 \\
550 & $23.3 \pm 2.8$ & $0.5 \pm 0.4$ & 2 & 4 \\
\hline
\end{tabular}

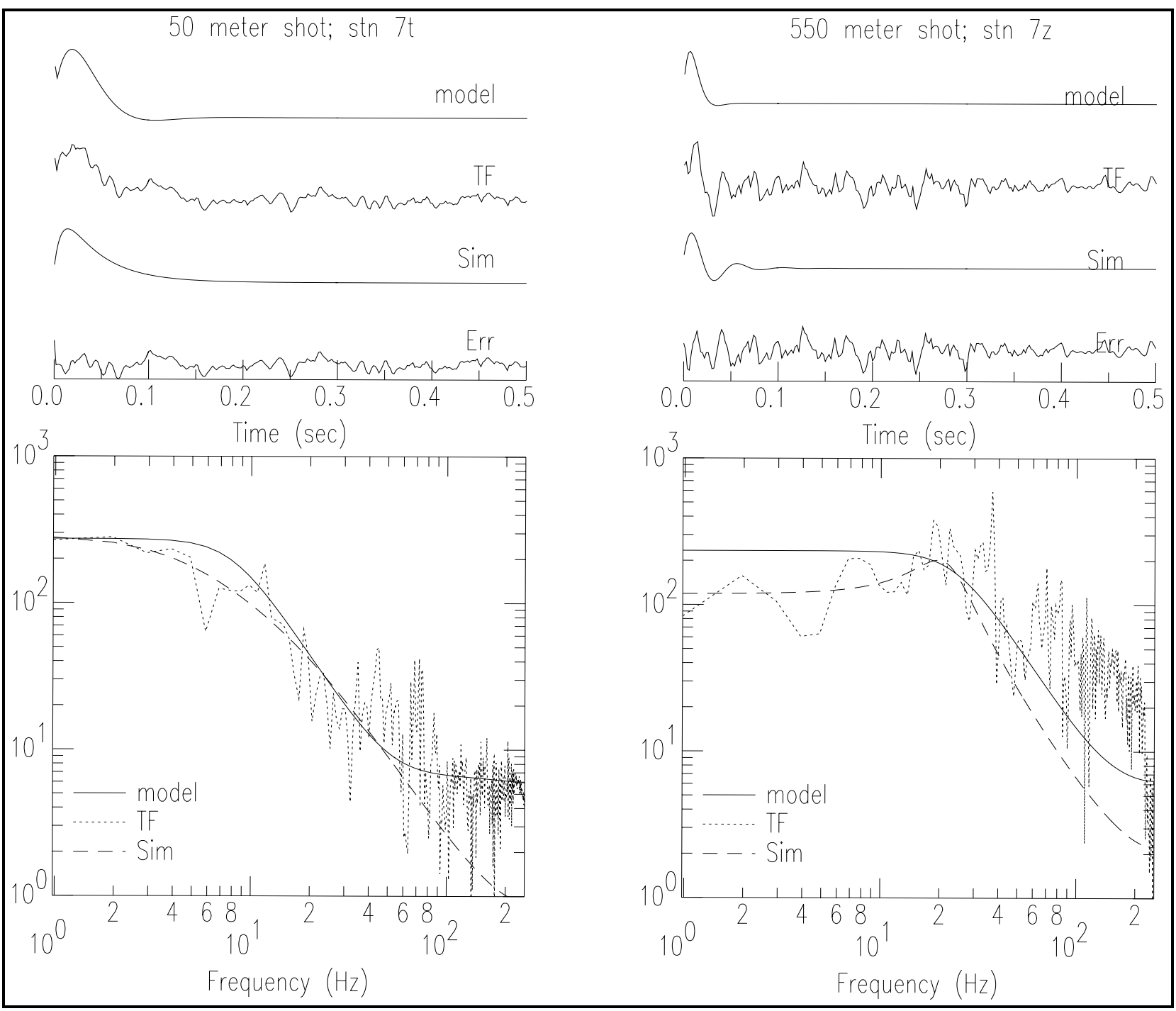

Figure 3. Effect of noise on parameter estimates. In the left-hand images, the noise causes the pole-zero analysis to overestimate the damping resulting in two real poles instead of a pair of complex conjugate ones. In the right-hand images, the analysis mistakenly interprets the noise as ringing in the signal and, consequently, underestimates the damping factor and the gain. Shown at the top of each frame are: (1) a model transfer function computed using the known yields, the mean corner frequencies given in Table 1 , and a damping factor of 0.7 ; (2) the experimental transfer function; (3) the model fit to the experimental data; and (4) the error between the data and the model. The spectra for 1,2 , and 3 are given in the graphs. 


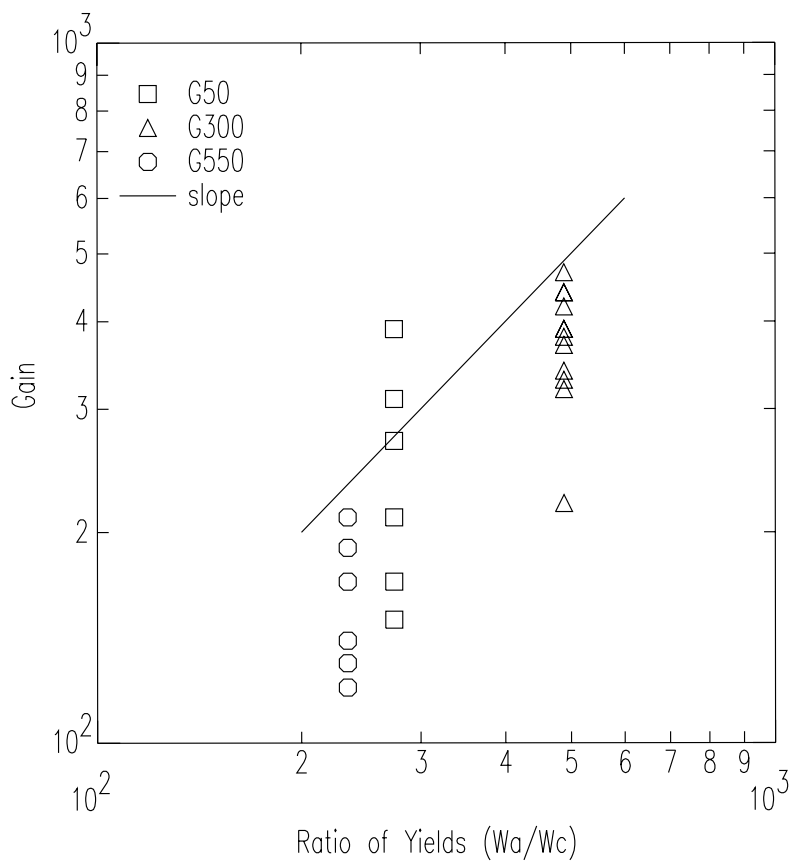

Figure 4. Experimental vs theoretical gain. The experimental gain was determined by calculating the spectral ratio between a 25 -ton signal and one obtained by transforming the calibration shot. The transformation was accomplished using a Butterworth filter with unit gain and a corner frequency determined from the mean corner frequencies of Table 1. The spectral ratios, although on the low side, are consistent with a linear dependence on the yield ratio.

To verify that the gains are consistent with cube-root scaling, the calibration shots were transformed to approximate the 25-ton shots by using a low-pass filter of unit gain, 0.7 damping, and the mean corner frequencies of Table 1 . The spectral ratios were then calculated between the real and the simulated data to determine the gains. In Fig. 4, the results are compared to the ratio of the charge weights, and are remarkably close to the theoretical result, $W_{l} / W_{c}$, for cuberoot scaling.

\section{Simplified Procedure}

To verify the pole-zero results, we employed a simplified optimization procedure. In the Goodman analysis, no constraints are applied. In this approach, Eq. (2) was calculated for an arbitrary set of frequencies, assuming the cube-root scaling, the known yield ratios, and damping of 0.7. These transfer functions were then applied to the calibration shots to simulate the large ones. The mean square errors between the data from large shots and the simulated data were then computed. The corner frequency that gave the minimum error was taken as the optimal solution. The results are given in Table 2 . This procedure gave unambiguous results for every set of signals; therefore, these mean values should be more reliable.

Table 2. Corner frequency.

\begin{tabular}{cccc}
\hline $\begin{array}{c}\text { Depth } \\
(\mathbf{m})\end{array}$ & $\begin{array}{c}\boldsymbol{f}_{0} \\
(\mathbf{H z})\end{array}$ & $\begin{array}{c}\text { No. of } \\
\text { stations }\end{array}$ & $\begin{array}{c}\text { No. of } \\
\text { components }\end{array}$ \\
\hline \hline 50 & $7.8 \pm 0.8$ & $\mathbf{2}$ & $\mathbf{6}$ \\
300 & $20.8 \pm 2.3$ & $\mathbf{4}$ & $\mathbf{1 2}$ \\
550 & $24.0 \pm 3.9$ & $\mathbf{2}$ & $\mathbf{6}$ \\
\hline
\end{tabular}

\section{Comparison of Results}

The results of both analyses are shown in Fig. 5, where the depth of burial is shown to have the expected significant effect on the corner frequency. Three data points are clearly too few to reliably determine the slope of the line, but it is clearly not inconsistent with either the slope of 0.42 given by Mueller-Murphy or that of 0.55 given by Denny-Johnson. Assuming source material properties of $2700 \mathrm{~kg} / \mathrm{m}^{3}$ for density and $6000 \mathrm{~m} / \mathrm{s}$ for compressional velocity, neither of

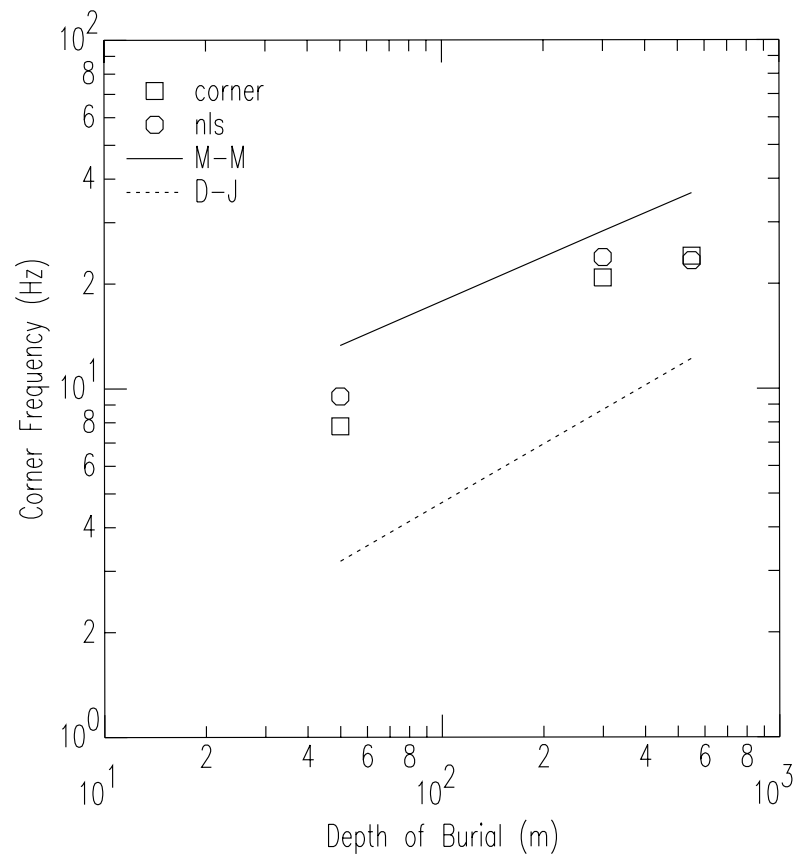

Figure 5. Comparison of results. The results from the two procedures to estimating the corner frequencies show a dependence on depth more or less consistent with previous observations. Here, they are compared to that predicted from the formulations of Mueller-Murphy and Denny-Johnson. 
the reference documents faired too well. The Mueller-Murphy formula overestimates the results by a factor of 1.4 to 1.7 , and the DennyJohnson formula underestimates them by a factor of 2 to 2.4 .

One expectation of the DOB experiment was that it would shed some light on the question of overshoot in the source function. Overshoot describes the degree of departure in Sharpe's model of the applied radial stress at the spherical boundary from a step function. In the pole-zero analysis, it can be appear as underdamping. As seen is Table 1, underdamping certainly occurred in some cases, especially at the greatest depths. Unfortunately, the variations in the estimates are large enough that the no clear departure from a step function in pressure can be clearly identified.

\section{Summary}

The depth of burial experiment clearly demonstrates the following:

- The simple Sharpe's model is an adequate representation of the seismic-source function for underground explosions.

- The source-function corner frequency for a contained explosion is highly dependent on depth of burial as previously found.

- The experimental data were consistent with the damping factor predicted by the Sharp model but the uncertainties were large.

The results also were found to be overpredicted by the Mueller-Murphy formula and underpredicted by that of Denny-Johnson.

\section{References}

M.D. Denny and D.M. Goodman (1990), “A Case Study of the Seismic Source Function: Salmon and Sterling Reevaluated," JGR 95(B12), 19, 705-19, 723.

M.D. Denny and L.R. Johnson (1991), "The Explosion Seismic Source Function: Models and Scaling Laws Reviewed," in Explosion Source Phenomenology, Taylor, Patton, and Richards, Eds., Geophysics Monograph 65, American Geophysical Union.

D.M. Goodman (1983), NLS: A System Identification Package for Transient Signals, Lawrence Livermore National Laboratory, Livermore, CA, UCID-19767.

R.A. Mueller and J.R. Murphy (1971), "Seismic Characteristics of Underground Nuclear Detonations," BSSA 61(6), 1675-1692.

W.S. Phillips, D.C. Pearson, C.L. Edwards, D.F. Baker, and M.D. Denny (1998), Near Source Recording of the Depth of Burial Experiment at Shagan River, Kazakstan: Data Report, Lawrence Livermore National Laboratory, Livermore, CA, UCRL-ID-131199.

J.A. Sharpe (1942), "The Production of Elastic Waves by Explosion Pressure. I. Theory and Empirical Field Observations," Geophysics $7(2)$. 